\title{
A new endophytic fungus CJAN1179 isolated from the Cholistan desert promotes lateral root growth in Arabidopsis and produces IAA through tryptophan-dependent pathway
}

\author{
Adeela Naureen ${ }^{1,3} \cdot$ Faiz-ul H. Nasim ${ }^{1,4}$ (1) Muhammad S. Choudhary ${ }^{2} \cdot$ Muhammad Ashraf $^{1}$. \\ Florian M. W. Grundler ${ }^{3}$ A. Sylvia S. Schleker ${ }^{3}$ (1)
}

Received: 11 July 2021 / Revised: 5 January 2022 / Accepted: 17 January 2022 / Published online: 17 February 2022

(C) The Author(s) 2022

\begin{abstract}
Fungi, important for growth of plants in arid lands, are expected to be involved in novel biochemical activities during fungal-plant interactions. We isolated 150 fungi associated with rhizosphere and root endosphere of two perennial grasses, Cymbopogon jwarancusa and Panicum antidotale, from Cholistan desert. The isolates were screened for their impact on plant growth and development using Arabidopsis thaliana (Col-0) as a model system. A root-endophytic fungus CJAN1179 from C. jwarancusa showed the highest plant growth-promoting effects. The most remarkable was enhanced number of lateral roots (3.1-fold). CJAN1179 produced indole-3-acetic acid (IAA) particularly in the presence of tryptophan. ITS sequence and phylogenetic analysis characterisation suggested the fungus to be a new species within Sordariomycetidae. CJAN1179 appears to promote plant growth by secreting IAA using tryptophan as a precursor. This fungus can be further explored for its suitability to promote growth of commercially important crops, particularly in arid regions.
\end{abstract}

Keywords Plant growth promotion · Cholistan desert · Cymbopogon jwarancusa · Root-associated fungi · Tryptophan . Auxin production

\section{Introduction}

Activities of a great variety of microorganisms including rhizospheric and endophytic fungi living in association with plants influence plant phenotype and development

Communicated by Olaf Kniemeyer.

A. Sylvia S. Schleker

sylvia.schleker@uni-bonn.de

Faiz-ul H. Nasim

faiz.nasim@iub.edu.pk; faiz.nasim@hotmail.com

1 Chemistry Department, The Islamia University of Bahawalpur, Bahawalpur 63000, Pakistan

2 Department of Botany, The Islamia University of Bahawalpur, Bahawalpur 63000, Pakistan

3 INRES, Molecular Phytomedicine, Rheinische Friedrich-Wilhelms-University Bonn, Karlrobert-Kreiten-Str. 13, 53115 Bonn, Germany

4 Institute of Biochemistry, Biotechnology and Bioinformatics, The Islamia University of Bahawalpur, Baghdad Ul Jadeed Campus, Bahawalpur 63000, Pakistan
(Rodriguez et al. 2009; Lugtenberg et al. 2013). These plant-associated microorganisms contribute to plant survival (Rodriguez et al. 2004; Ortíz-Castro et al. 2009). Plants growing and surviving in habitats with extreme climatic conditions such as arid soils develop functional diversity and the root-associated microbial populations must be contributing their important role here (Zuo et al. 2015). Deserts are considered to be among the habitats where plants are exposed to extreme environmental conditions such as drought, high temperature, extreme $\mathrm{pH}$ and salinity. Root-associated microbial communities of desert plants have been suggested as an important factor responsible for adaptation of desert plants to the harsh desert environment (Bhatnagar and Bhatnagar 2005; Cherif et al. 2015; Makhalanyane et al. 2015). It is, therefore, of high interest to explore the diversity and role of microorganisms originating from desert plants for plant growth and survival. The Cholistan desert located in the southern part of Bahawalpur in Punjab, Pakistan is a largely unexplored habitat in this respect (Rafay et al. 2013). Its flora is composed of xerophytic species that have adapted to the desert conditions (Arshad et al. 2008; Hameed et al. 2011). Unique microbial communities producing novel 
metabolites involved in beneficial fungus-plant interactions are expected to reside in association with the roots of plants growing in this desert.

Fungi are a group of diverse organisms with multiple metabolic activities and a large group of endophytic fungi establishes mycorrhizal or non-mycorrhizal associations with the plant roots (Rodriguez et al. 2009). Endophytic fungi have been reported to mediate a number of beneficial roles inside plants (Rodriguez et al. 2004; Gond et al. 2010; Khan et al. 2012; Zhou et al. 2014) including involvement of endophytes in plant nutrient uptake and the production of secondary metabolites to stimulate plant growth (Waqas et al. 2012). However, the mechanistic biochemical details of endophyte-plant associations are often not clearly understood (Dovana et al. 2015).

When fungi and plant roots sense the presence of each other in rhizosphere, they produce certain metabolites to initiate a mutualistic fungus-plant interaction that is well organised and bidirectional. Endophytes absorb required nutrients from plant roots to produce metabolites that directly influence plant growth promotion or indirectly by initiating plant-signalling mechanisms leading to the production of growth-promoting molecules, especially phytohormones (Ortíz-Castro et al. 2009). A number of plant beneficial fungi have been reported to produce phytohormones (Aloni et al. 2006; Hamayun et al. 2010). Microbial phytohormones, e.g. auxins, cytokinins and gibberellins are either involved in plant growth promotion or help plant cope with biotic or abiotic stress. Thus, root-associated plant beneficial fungi can be explored as an environment-friendly tool to promote plant growth and ensure high productivity (Rodriguez et al. 2009; Mei and Flinn 2010).

Towards exploring the diversity and beneficial properties of cultivable desert plant root-associated fungi, we collected 150 fungal isolates from the rhizosphere and endosphere of Cymbopogon jwarancusa and Panicum antidotale, two perennial grasses commonly growing in the Cholistan desert. We screened these fungal isolates for their impact on growth and development using A. thaliana as a model. The knowledge of plant root-associated fungi and their beneficial roles during interaction with $A$. thaliana has provided insights into plant-microbe associations and is expected to identify microorganisms suitable for use in sustainable agricultural management strategies.

\section{Materials and methods}

\section{Sample collection}

Roots with attached soil from three ecotypes of $P$. antidotale P1 (accession \# PA-YZ-PL14-18), P4 (accession \# PA-YZ-PL02-37) and P10 (accession \# PA-YZ-PLH-28) and two ecotypes of $C$. jwarancusa $\mathrm{C} 10$ (accession \# CHMW-PL10-13) and C12 (accession \# CH-NT-PL12-16) were collected on 14 March 2013 in sterile plastic jars from the Cholistan Institute of Desert Studies (CIDS) located between $27^{\circ} 42^{\prime}$ and $29^{\circ} 45^{\prime} \mathrm{N}$ latitude and $69^{\circ} 52^{\prime}$ and $75^{\circ} 24^{\prime} \mathrm{E}$ longitude, the Islamia University of Bahawalpur (IUB), Pakistan. Collected samples were stored in a cool and dark place until further processing.

\section{Isolation of fungi from rhizospheric soils}

Rhizospheric soil of each sample was suspended in $30 \mathrm{ml}$ sterile Ringer solution. $100 \mu \mathrm{l}$ of the optimal dilution of this solution suitable to obtain separated fungal colonies, was spread on Sabouraud Agar (SA) $(\mathrm{pH}=6.8)$ plates including Streptomycin and Penicillin (100 and $50 \mathrm{mg} / \mathrm{L}$, respectively) to inhibit bacterial growth (Sun et al. 2009). The plates were incubated in inverted position at $37{ }^{\circ} \mathrm{C}$ until fungal colonies appeared. As negative control $100 \mu \mathrm{l}$ sterile Ringer solution was spread on SA plates in parallel. A liquid culture of each fungal isolate was grown in Sabouraud's Broth, (SB) $\mathrm{pH}=6.8$ at $37^{\circ} \mathrm{C}$ and $250 \mathrm{rpm}$. Fungal isolates were initially characterised on the basis of morphology. Duplication was avoided. The fungal spores were stored in 50\% SB glycerol at $-70{ }^{\circ} \mathrm{C}$.

\section{Isolation of root-endophytic fungi}

To isolate root endophytes, roots were surface sterilised using an earlier described method (Ahmed et al. 2012) with a few modifications. All attached soil was thoroughly washed off using tap water. The roots were then rinsed stepwise with $70 \%$ ethanol for $30 \mathrm{~s}, 0.01 \% \mathrm{HgCl}_{2}$ for $5 \mathrm{~min}, 0.5 \%$ $\mathrm{NaOCl}$ for 3 min followed by washing with sterile water to remove chemicals. These roots were dried between folds of sterile filter paper and cut into small pieces of about $8 \mathrm{~mm}$ that were placed on SA plates for fungal isolation. The plates were incubated as describes above. The water from last wash before drying the roots was plated on SA plates as negative control (Fouda et al. 2015). Spores of isolated endophytic fungi were stored as stated earlier.

\section{Screening fungal isolates for their impact on plant development}

The impact of 150 fungal isolates on growth and development of wild-type Arabidopsis thaliana Columbia-O (Col-O) was tested in fungus-plant interaction assays performed as follows. Freshly grown purified fungal colonies on SA plates were used for fungus-plant interaction assays.

A. thaliana seeds were sterilised through slightly modified standard method (Sijmons et al. 1991; Bohlmann and Wieczorek 2015). In brief, seeds were placed in $0.6 \% \mathrm{NaOCl}$ 
for $5 \mathrm{~min}$. The supernatant was removed and the seeds were soaked in $70 \%$ ethanol for 5 min followed by washing them 5 times with sterile water and drying in between the layers of sterile filter paper. Surface sterilised dried seeds were stored at $4{ }^{\circ} \mathrm{C}$ until use. For the interaction assays, two sterile seeds were placed $4 \mathrm{~cm}$ apart and at $1.5 \mathrm{~cm}$ from the upper edge of the plate on $2 \%$ Knop agar plates that were incubated in tilted position in a growth chamber for 6 days $\left(23 \pm 1{ }^{\circ} \mathrm{C}, 16: 8\right.$, light/dark, photoperiod, light intensity of $150 \mu \mathrm{mol} \mathrm{m} \mathrm{m}^{-2} \mathrm{~s}^{-2}$ of PAR). To determine the impact of the fungal isolates on plant growth and to allow proper comparison, all the plants used for the interaction assay had to fulfil the following criteria on day 6: primary root length of almost $2.5 \mathrm{~cm}$, rosette diameter of about $0.8 \mathrm{~cm}$ and 4 leaves.

A fungal plug of $5 \mathrm{~mm}$ diameter was placed on the plate at $5 \mathrm{~cm}$ distance from the root end of the 6-day-old plants. These plates along with control plates (plants without fungal exposure) were incubated in a growth chamber as described above.

Parameters related to plant growth including primary root length $(\mathrm{cm})$, number of lateral roots, root hairs, number of leaves, rosette diameter $(\mathrm{cm})$, leaf colour, fresh shoot weight $(\mathrm{g})$, flower stalk length $(\mathrm{cm})$ and flower blossom status were recorded during the assay at specific intervals. The first measurement of these parameters was done at the time of fungal inoculation, 0 days' post-inoculation (dpi). Subsequent measurements were performed at equal intervals of 4 days up to 16 dpi. Among all parameters, root hairs, leaf colour and flower blossom were qualitative parameters and determined visually. The initial screening of all 150 fungal isolates for their impact on plant development was done with 2 plants per fungal isolate on Knop agar plate.

\section{Detailed analysis of the impact of selected fungal isolates on plant development}

Out of 150 fungi, a root endophyte CJAN1179 obtained from C. jwarancusa possessed the most remarkable beneficial effect on plant root exhibited as highly enhanced number of lateral roots and little effect on shoot growth. Isolates PAAN1181 and PAAN1135 obtained from P. antidotale exhibited the worst negative effects and almost no effect on plant growth, respectively.

To validate the observed plant growth-promoting properties of CJAN1179, a detailed analysis was performed using PAAN1181 and PAAN1135 as controls in the presence or in the absence of CJAN1 179 for comparison. The fungus-plant interaction assay was done in 3 biological replicates with 10 technical replicates each (30 plants each for each variant in total). The assay was done as described above with the exception that besides fungal plug inoculation (FPI), a second fungus-plant interaction assay setup using fungal culture filtrates was performed. FPI was done using 6-day-old plants fulfilling the criteria described above (Ortíz-Castro et al. 2009). Fungal culture filtrate inoculation (FCFI) was done with 9-day-old plants with the following growth status: root length around $5 \mathrm{~cm}$, number of lateral roots almost 10 , number of leaves 6 and rosette diameter almost $2 \mathrm{~cm}$. At this time point, the roots attain enough root mass to absorb culture filtrate (Daneshkhah et al. 2013).

For the FCFI assay, liquid cultures of the three fungi were grown in $\mathrm{SB}$ at $37{ }^{\circ} \mathrm{C}$ on a rotary incubator at $250 \mathrm{rpm}$ for 14 days. Each culture was filtered through $0.2 \mu \mathrm{m}$ membrane syringe filter. Fungal culture filtrates and control $(150 \mu \mathrm{l})$ were directly applied onto the plant roots and left for half an hour allowing plant roots to absorb the filtrate. The treated plants were incubated in a growth chamber. The parameters to document plant development were recorded as described above.

\section{Statistical analysis}

The obtained data were analysed using SPSS 15. Plant growth parameters were taken as dependent variable. Fungal inoculations (FPIs/FCFIs) and dpi were taken as fixed factors or independent variables. Some plant growth parameters were analysed with two fixed factors (fungal inoculations and dpi) through descriptive statistics and significance $(P<0.05)$ using "Univariant Analysis of Variance". Parameters measured at only 16 dpi were analysed with only one fixed factor (fungal inoculations) using "One way ANOVA". Least significant difference (LSD) between control plant and inoculated plant growth parameters were calculated based on observed means through "Post Hoc Tests". The mean differences were categorised as significant on the basis of the size of effect as: very significant $P^{* *}(P \leq 0.01)$, significant $P^{*}(P \leq 0.05)$ and non-significant $P^{\mathrm{NS}}(P>0.05)$. All quantitative plant growth parameters were statistically analysed. Root hairs, colour of leaves and flower blossom were not statistically evaluated because these are qualitative parameters.

\section{Detection of auxin (IAA) production}

Salkowski colorimetric assay was used to determine whether CJAN1179 produces indole-3-acetic acid (IAA), a predominant naturally occurring auxin (Glickmann and Dessaux 1995). This assay is the most recommended method for the detection of IAA production in microbial cultures (Sarwar and Kremer 1995; Mohite 2013; Rehman et al. 2015).

A standard curve (absorbance vs. concentrations) was prepared by mixing different amounts of a $0.01 \%$ IAA solution $(0 \mu \mathrm{l}, 5 \mu \mathrm{l}, \ldots 30 \mu \mathrm{l}), 100 \mu \mathrm{l}$ Salkowski reagent and $\mathrm{ddH}_{2} \mathrm{O}$ up to a total volume of $250 \mu \mathrm{l}$ in triplicate. After 20 min of incubation at $26^{\circ} \mathrm{C}$, the OD was measured at $530 \mathrm{~nm}$ using a plate reader. 
The three fungi (CJAN1179, PAAN1135 and PAAN1181) were cultured in SB with and without $1 \%$ L-tryptophan using the same culture conditions. The reaction mixture $(250 \mu \mathrm{l})$ containing different pre-determined volumes of fungal culture filtrates (CFs), viz., CJAN1179, PAAN1135 and PAAN1181 CF with and without tryptophan, and IAA controls were prepared in triplicate and handled as described above. IAA concentration of each sample was calculated using the standard curve.

\section{Identification of beneficial fungus CJAN1179}

\section{Molecular characterisation}

Genomic DNA of CJAN1179 was isolated with some modifications in already reported method (Liu et al. 2000). $25 \mathrm{mg}$ of freshly grown colony of CJAN1179 SA was taken into a 2-ml tube containing stainless steel beads of $5 \mathrm{~mm}$ diameter and $500 \mu \mathrm{l}$ lysis buffer (400 mM Tris-HCl, $60 \mathrm{mM}$ EDTA, $150 \mathrm{mM} \mathrm{NaCl}$ and $1 \%$ SDS, $\mathrm{pH}=8.0$ ). The tube was left at room temperature for $5 \mathrm{~min}$. To this solution, $150 \mu \mathrm{l}$ of potassium acetate solution $(60 \mathrm{ml} 5 \mathrm{M}$ potassium acetate, $11.5 \mathrm{ml}$

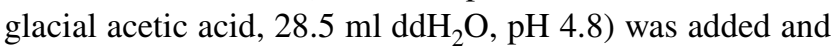
the tube was placed in Tissue Lyser for lysis of fungal colony at $40 \mathrm{~Hz}$ for $10 \mathrm{~min}$. The tube was then placed at $-30{ }^{\circ} \mathrm{C}$ for $20 \mathrm{~min}$ and centrifuged at $17,135 \times g$ for $5 \mathrm{~min}$. Supernatant from the tube was transferred to another tube and extracted twice with equal volumes of chloroform: isoamyl alcohol (24:1) before centrifugation. The supernatant was then mixed with an equal volume of isopropanol. The tube was placed at $4{ }^{\circ} \mathrm{C}$ for $1 \mathrm{~h}$ and centrifuged at $17,135 \times g$ for $5 \mathrm{~min}$. The DNA pellet was washed 3 times with $300 \mu \mathrm{l} 70 \%$ ethanol, air dried and dissolved in $50 \mu \mathrm{l}$ of $1 \mathrm{x}$ Tris EDTA. Integrity of the extracted DNA was verified by agarose gel electrophoresis.

For amplification, $10 \mathrm{ng}$ fungal DNA was used as template in a standard PCR reaction containing $10 \mathrm{mM} 1 \mathrm{X}$ PCR buffer, $1.5 \mathrm{mM} \mathrm{MgCl} 2,100 \mathrm{mM}$ of each of the four dNTPs, $1.25 \mathrm{U}$ Taq DNA polymerase and $10 \mathrm{pmol}$ of each of the two primers ITS1 and ITS4, forward and reverse primers, respectively. Amplification conditions were as follows: initial denaturation at $94{ }^{\circ} \mathrm{C}$ for $5 \mathrm{~min}, 30$ cycles of $94^{\circ} \mathrm{C}$ for $30 \mathrm{~s}, 1 \mathrm{~min}$ at $50^{\circ} \mathrm{C}$ and $1 \mathrm{~min}$ at $72{ }^{\circ} \mathrm{C}$, followed by final extension at $72{ }^{\circ} \mathrm{C}$ for $10 \mathrm{~min}$. Expected size of the PCR product was verified by agarose gel electrophoresis. The DNA fragment was cleaned, purified and sent to GATC Biotech, Cologne Germany for sequencing.

\section{Phylogenetic analysis}

For phylogenetic lineage determination of CJAN1179, the route of analysis followed was adopted from a published description with some modifications (Nasim et al. 2018).

(a) Multiple sequence alignment of ITS sequences from query and selected fungal species: NCBI BLAST was used to reveal the number of maximum hits for the query sequence and to retrieve the most similar sequences for construction of the phylogenetic tree (Altschul et al. 1997). Genious bioinformatics software was used to perform multiple sequence alignment of our query along with selected sequences (Kearse et al. 2012). In multiple sequence alignment, truncated sequences were deleted and longer sequences were shortened to make them all equal in length.

(b) Molecular phylogenetic analysis of ITS for query along with closely related fungal species: Neighbour-joining method (Saitou and Nei 1987) was used to infer the evolutionary history. Evolutionary history of the taxa analysed was represented using the bootstrap consensus tree inferred from 100 replicates. Branches corresponding to partitions reproduced in less than $50 \%$ bootstrap replicates were collapsed. The percentage of replicate trees in which the associated taxa clustered together in the bootstrap test (100 replicates) are shown next to the branches (Felsenstein 2005). The tree was drawn to scale, with branch lengths in the same units as those of the evolutionary distances used to infer the phylogenetic tree. P-distance method (Nei and Kumar 2000) was used to compute the evolutionary distances which are in the units of the number of base differences per site. The analysis involved 38 nucleotide sequences. All positions containing gaps and missing data were eliminated. A total of 429 positions in the final dataset were available in this analysis. MEGA5 (Tamura et al. 2011) was used to conduct evolutionary analyses.

\section{Morphological characterisation}

After phylogenetic analysis of the fungal sequence on the basis of ITS regions using molecular and phylogenetic tools, the potential fungus CJAN1179 was grown both on SA plates and in SB medium for possible macroscopic studies, i.e. shape and colour of fungal colony and spores to establish further placement of fungus in taxonomic lineage. Detailed microscopic studies were not performed as molecular tools give more precise taxonomic information and are preferred over conventional morphological parameters for identification of the fungus (Tarbell 2008) and ITS region is considered the most suitable DNA barcode for fungi (Schoch et al. 2012).

\section{Results}

\section{Isolation of fungi}

In total, 150 morphologically different fungi were isolated. Among those, 79 (48 rhizospheric and 31 root endophytes) fungi were isolated from three ecotypes of $P$. antidotale, 
whereas 71 (56 rhizospheric and 15 root endophytes) fungi were isolated from two ecotypes of $C$. jwarancusa.

\section{Root endophyte CJAN1179 is a plant growth-promoting fungus}

Impact of all the 150 fungal isolates on the growth and development of A. thaliana Col-0 was investigated using an in vitro fungus-plant interaction assay. Among all tested isolates, CJAN1179, a root endophyte from $C$. jwarancusa, influenced plant growth most positively (Fig. 1). In the presence of CJAN1179, the number of $A$. thaliana lateral roots increased significantly and a little increase in root hairs and shoot weight was noticed. For all other plant growth parameters, CJAN1179 caused negligible or no effects. On the opposite, isolate PAAN1181 was the most detrimental for the plant and isolate PAAN1135 appeared to show no effect in this regard (Fig. 1, Suppl. Fig. 1). In the subsequent detailed characterisation of CJAN1179 with respect to plant growth-promoting effects and possible phytohormone production, PAAN1181 and PAAN1135 were included in the experiments for comparison.

\section{The living fungus, not the culture filtrate mediates plant growth promotion}

To figure out whether the fungal impact on plant development is mediated by fungal metabolites of the culture filtrate or requires the interaction of the actively growing fungus with the plant roots, effects of the three fungi on plant growth were compared between two types of inoculations FPI (living fungus) and FCFI (culture filtrate). The two treatments were found to have obvious differences (Suppl. Fig. 2).

CJAN1179 plug inoculation promoted plant growth most remarkably with regard to the number of lateral roots $(210 \%$ increase), and showed little effect on root hairs (qualitative parameter) and shoot weight (33.3\% increase) as compared to the corresponding controls but showed little or no effect

Fig. 1 Excessive lateral root formation induced by CJAN1179 in A. thaliana as compared to unexposed control plants and plants exposed to PAAN1135 and PAAN1181 at equal intervals of 4 days up to $16 \mathrm{dpi}$. $C$ control plants, CJAN1179 fungus with positive effect on plants, PAAN1135 fungus with no effect on plants, PAAN1181 fungus with negative effect on plants, $d p i$ day post-inoculation

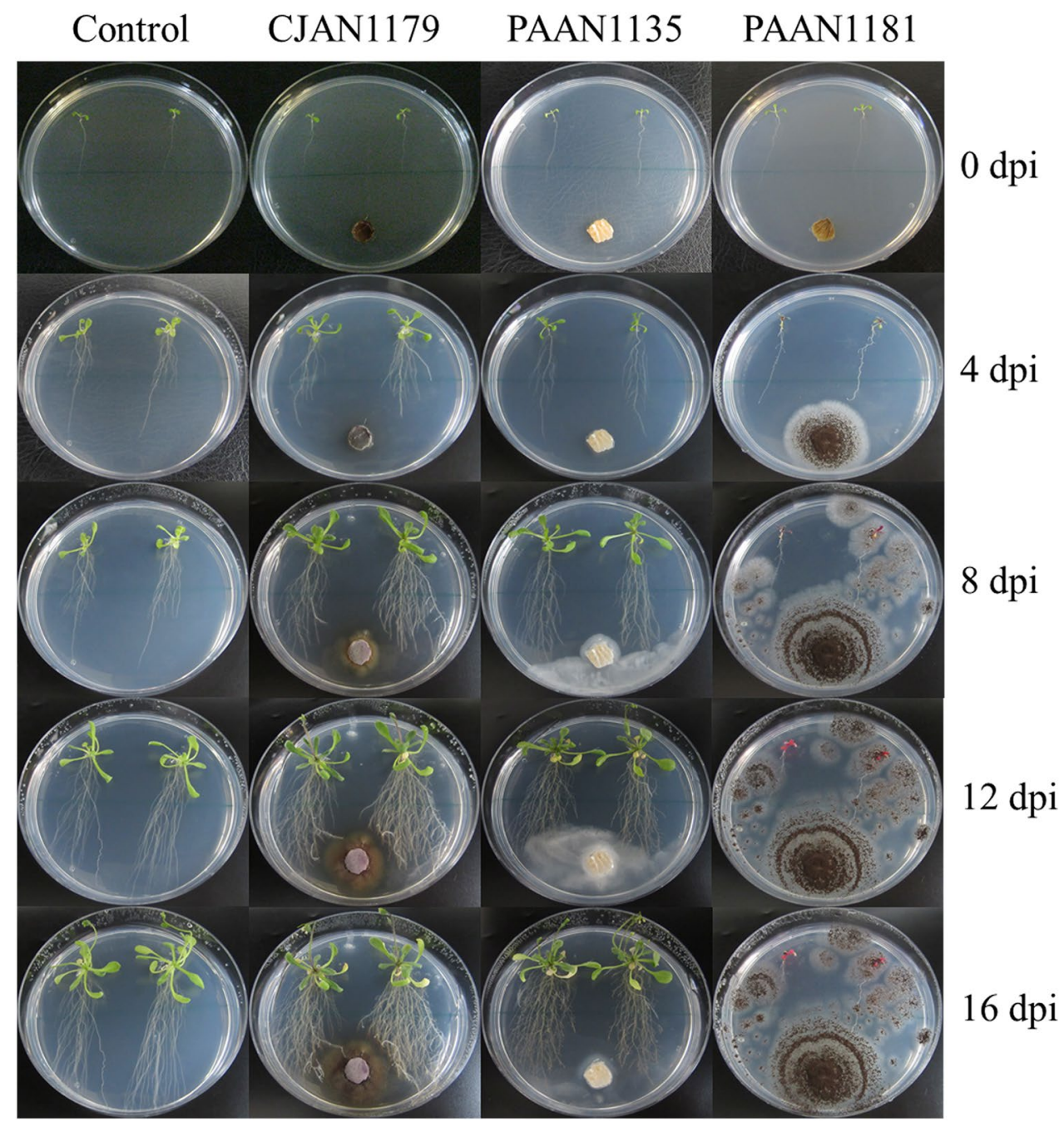


on other growth parameters. Final increase in the number of lateral roots in plants exposed to CJAN1179 CF (9.1\%) as compared to that of the controls was negligible when compared to that in the case of CJAN1179 plug inoculation (Fig. 2).

PAAN1181 plug inoculation caused highly negative effects on all root, shoot and flower growth (no flowers appeared in this case) parameters. In the same way, PAAN1181 CF application showed very less drastic effects on plant growth as compared to that in case of plug inoculation. PAAN1135 plug as well as CF inoculations did not mediate any change in almost all plant growth parameters with the exception that the plant leaves were yellowish at 16 dpi after PAAN1135 plug inoculation (Suppl. Fig. 2).

These observations reveal that when the model plant roots come in contact with the fungus CJAN1179, the influence on plant growth is many times greater than when the culture filtrate of the same fungus is used. An obvious increase in the number of lateral roots was observed from 0 to $16 \mathrm{dpi}$ with the culture plug (FPI), however, when the culture filtrate $(\mathrm{CF})$ was applied, a change in lateral root number was noticed only at $4 \mathrm{dpi}$, which thereafter became practically steady until 16 dpi.

\section{CJAN1179 and PAAN1181 showed statistically significant effects on plant growth}

Effects of the three fungi on A. thaliana growth in reference to quantitative parameters were statistically analysed. CJAN1179 plug inoculation showed very significant $P^{* *}$ $(P<0.01)$ positive effects on the number of lateral roots, number of leaves, rosette diameter and shoot weight in these parameters but did not show any effect $P^{\mathrm{NS}}(P>0.05)$ on other growth parameters, e.g. length of primary root $(P=0.133)$ and flower stalk length $(P=0.149)$. CJAN1179 CF showed significant positive effect $P^{*}(P<0.05)$ only on number of lateral roots $(P=0.020)$ and number of leaves $(P=0.021)$ and did not show any effect $P^{\mathrm{NS}}(P>0.05)$ on other growth parameters. In contrast, PAAN1181 plug inoculation caused very significant $P^{* *}(P<0.01)$ negative effects on all root, shoot and flower growth parameters. PAAN1181 CF caused significant negative effects $P^{*}(P<0.05)$ on plant growth whereas PAAN1135 plug as well as $\mathrm{CF}$ inoculation did not show any significant change $P^{\mathrm{NS}}(P>0.05)$ in plant growth.

In statistical analysis, mean differences between inoculated and control plants for various plant growth metrics indicate the extent or intensity of fungal effect on plant growth, which can be seen as differences between the lines and bars of graphs. Thus, according to these mean differences, CJAN1179 had a significant favourable effect only on number of lateral roots when active fungal plug inoculation was used (Fig. 2).

\section{CJAN1179 produces IAA}

The most remarkable beneficial effect of CJAN1179 on plant growth was found to be a significant increase in the number of lateral roots. The phytohormone auxin, most predominantly occurring as IAA, is an important player in the formation of lateral roots (Idris et al. 2007). Thus, we

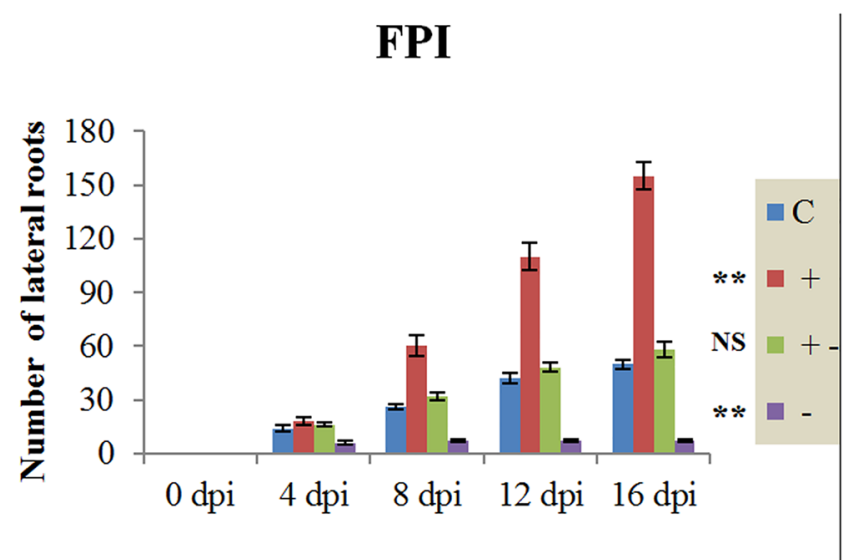

Fig. 2 The most remarkable plant growth in the form of enhanced lateral root growth in case of direct CJAN1179-A. thaliana root interaction through FPI as compared to that of FCFI effect on lateral roots: FPI = fungal plug inoculation, FCFI fungal culture filtrate inoculation, $d p i$ days post-inoculation, $C$ control plants (plants without any fungal exposure), $+=$ CJAN1179 (fungus with positive effect on plants), $+-=$ PAAN1135 (fungus with no effect on plants),

\section{FCFI}

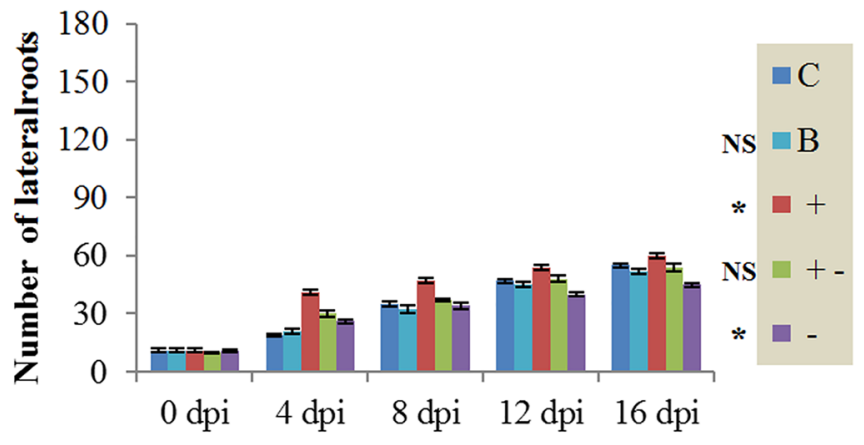

- =PAAN1181 (fungus with negative effect on plants). The mean differences between inoculated plants and control plants; very significant $\left(P^{* *}\right)$ at $P \leq 0.01$, significant $\left(\mathrm{P}^{*}\right)$ at $P \leq 0.05$ and non-significant $\left(\mathrm{P}^{\mathrm{NS}}\right)$ at $P>0.05$ (shown with the legend). Mean \pm SE of three independent experiments, $n=30$. A complete comparison of effects of CJAN1179 on plant root, shoot and flower growth between FPI and FCFI is given in Suppl. Fig. 2 
hypothesised that CJAN1179 is generating its plant growth promotion effect through production and secretion of IAA.

The presence of IAA in the CF of the three fungi was detected using Salkowski's test. During this test, pure IAA results in a pink colour but red colour indicates the production of tryptophol (TOL) along with IAA from tryptophan (Robinson et al. 1998; Rahman et al. 2010). TOL has been reported as a by-product formed during the conversion of L-tryptophan to IAA through the indole-3-pyruvate pathway of tryptophan-dependent production of IAA and is convertible to IAA (Furukawa et al. 1996; Maor et al. 2004). The reaction mixture containing CJAN1179 CF grown in the absence of tryptophan showed no colour change indicating that there is no or very low IAA production in the absence of tryptophan. In case of CJAN1 179 cultured in the presence of tryptophan, the CF changed colour of the Salkowski's reagent to red. This shows that CJAN1179 produces IAA and possibly TOL when grown in the presence of tryptophan.

Evaluation using colourimetric assay at $530 \mathrm{~nm}$ revealed that in the absence of tryptophan, CJAN1179 only was able to generate extremely modest levels of IAA. That is why no significant colour change was visible.

However, CJAN1179 was able to produce a lot of IAA $(1638 \mu \mathrm{g} / \mathrm{ml})$ in the presence of tryptophan.

Under similar conditions, PAAN1135 and PAAN1181 produced very low IAA amounts of 304 and $172 \mu \mathrm{g} / \mathrm{ml}$, respectively, and that only in the presence of tryptophan (Fig. 3).

\section{CJAN1179 belongs to the Sordariomycetidae}

\section{Molecular characterisation and phylogenetic analysis of CJAN1179}

The obtained ITS sequence of CJAN1179 is available at NCBI, accession number LC198062. The phylogenetic tree is representing 3 different clades (monophyletic groups), i.e. Magnaporthe oryzae, Magnaporthe grisea and uncultured fungi (Fig. 4). Bootstrap values are supporting the analysis. Using BLAST, the query sequence was found to match best with the corresponding sequences of four uncultured fungi with the GenBank accession nos. AJ875356, FN397168, GU910815 (Sordariales) and GQ924058 (Diaporthales) (Suppl. Fig. 3). Query coverage is $100 \%$ with $1 \%$ gaps and sequence identity between 92 and $94 \%$.

\section{Morphological characterisation}

Following a molecular phylogenetic analysis of CJAN1179's ITS sequence for taxonomic lineage identification, a fungal colony produced on SA plate and spores or fruiting bodies

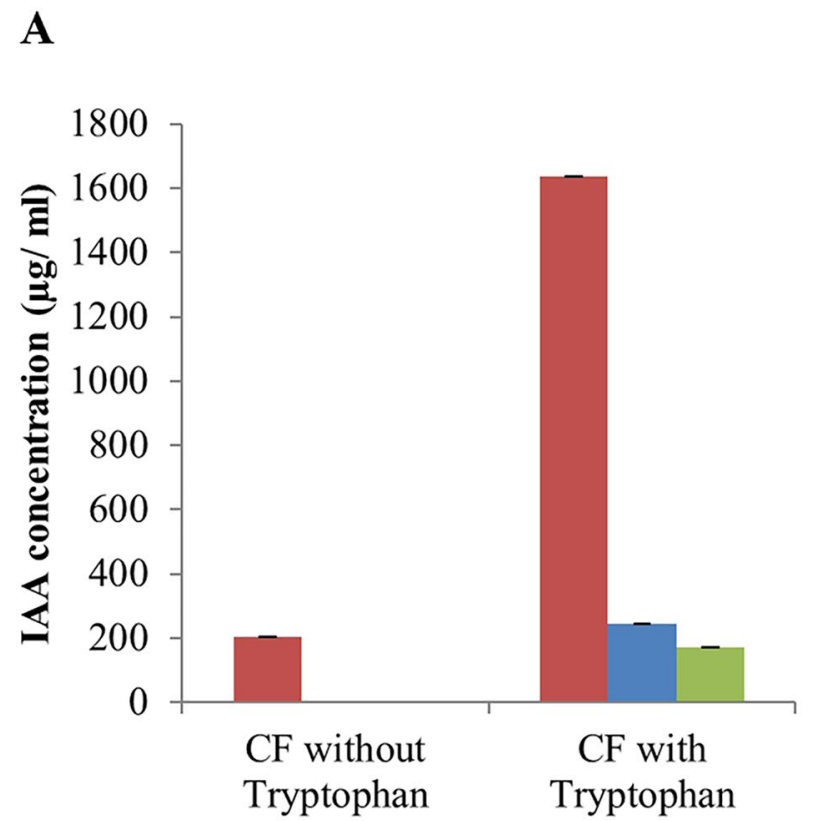

Fig. 3 A IAA concentration of the culture supernatant of the three fungi grown with and without tryptophan. $C F$ fungal culture filtrate. Using OD values of fungal culture filtrates, concentration of auxin was calculated through standard curve formula $\mathrm{X}=\mathrm{Y}+0.010 / 0.018$ ( $\mathrm{X}=$ conc. of IAA to be calculated, $\mathrm{Y}=$ mean $\pm \mathrm{SE}$ of three replicates of OD values for each assay in triplicate, $n=9$ ), $\mathbf{B}$ colour change
B

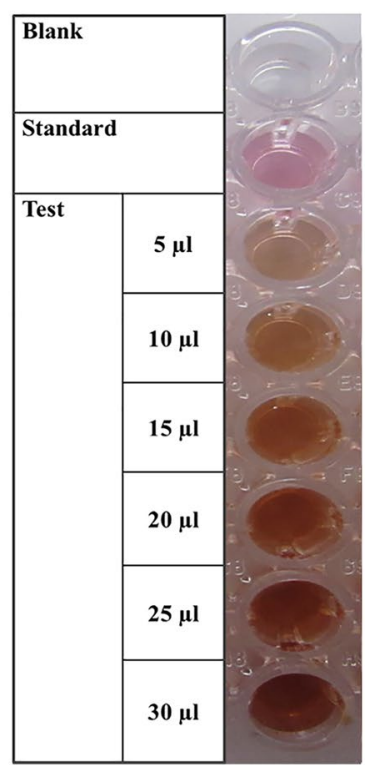

in reaction mixture having CJAN1179 $\mathrm{CF}$ with tryptophan after incubation, Blank = reaction mixture without standard or test sample, Standard $=0.01 \%$ Indole- 3 -acetic acid solution, Test $=$ reaction mixture having different volumes (5-30 $\mu \mathrm{l})$ of sample (CJAN1179 $\mathrm{CF}$ with $0.1 \%$ L-tryptophan). Reaction mixture $=$ Test/ standard/ blank $+\mathrm{H}_{2} \mathrm{O}+$ Salkowski reagent $=250 \mu \mathrm{l}$ 


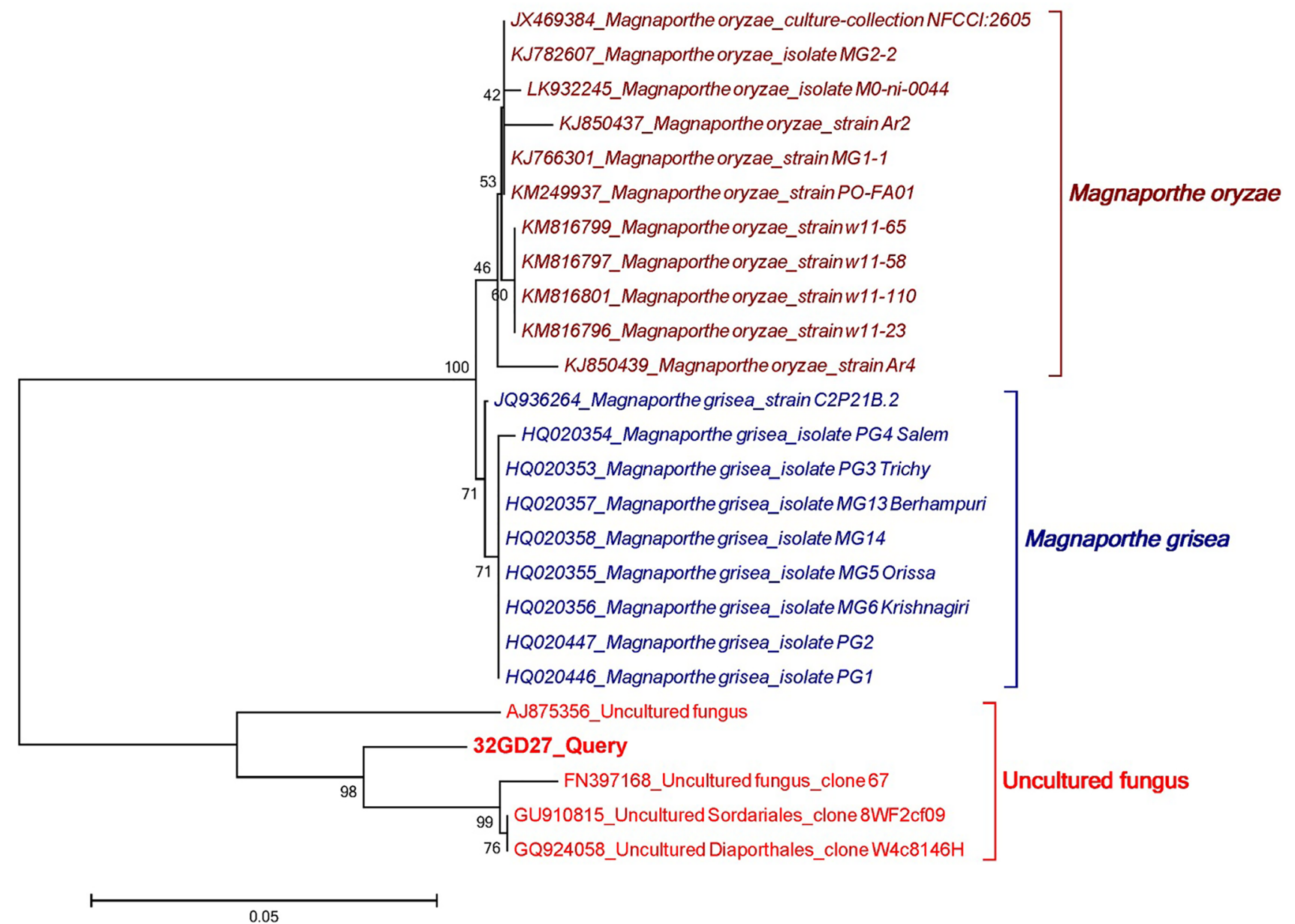

Fig. 4 Phylogenetic comparison of CJAN1179 (32GD27_Query) ITS sequence with corresponding sequences of selected fungal species: The query sequence is clearly separated from the clades of Mag-

developed in SB media were subjected to a macroscopic examination. CJAN1179 produced a yellowish-brown mycelial colony and turned SB to a deep cherry red coloured medium indicating the secretion of colourful metabolites. The fungus produces smooth, hairy black spherical sporocarps in SB. The fully mature sporocarps appear to be flask-shaped with a beak-like structure. These sporocarps can generate a fully grown fungal colony on SA medium. A sporocarp first transforms into a little yellowish-brown fungal mycelia colony, which then matures into a purplishgrey colony, turning the agar medium cherry red (Fig. 5).

\section{Discussion}

Because of plant-microbe interactions, the plant-soil interface is thought to be a hotbed of biological activity (Morgan et al. 2005). Plants thriving in a variety of environmental settings including deserts appear to be linked with endophytic naporthe oryzae and Magnaporthe grisea and fall into the clade of four uncultured fungi, two of them are unclassified and other two are uncultured Sordariales and uncultured Diaporthales

and rhizospheric microorganisms that are thought to be involved in a number of biological processes enabling these plants to survive in harsh climates (Cherif et al. 2015; Makhalanyane et al. 2015). Below-ground microflora, which includes plant growth-promoting fungi (PGPF), is, therefore, critical for establishment of vegetation and a focus for investigators exploring sustainable development strategies. Keeping this hypothesis in mind, we have isolated rhizospheric and root-endophytic fungi from plants growing in the Cholistan desert and screened them for their plant growthpromoting potential. Using A thaliana as a model plant, we investigated plant growth-promoting features of 150 fungal isolates and identified an extremely effective PGP fungus CJAN1179. When compared to unexposed plants, this fungus has a considerable impact on root formation and increased the number of lateral roots many folds.

A variety of phytohormones, such as auxin, cytokinins and gibberellins, are involved in different aspects of plant growth (El-Showk et al. 2013). They are produced 

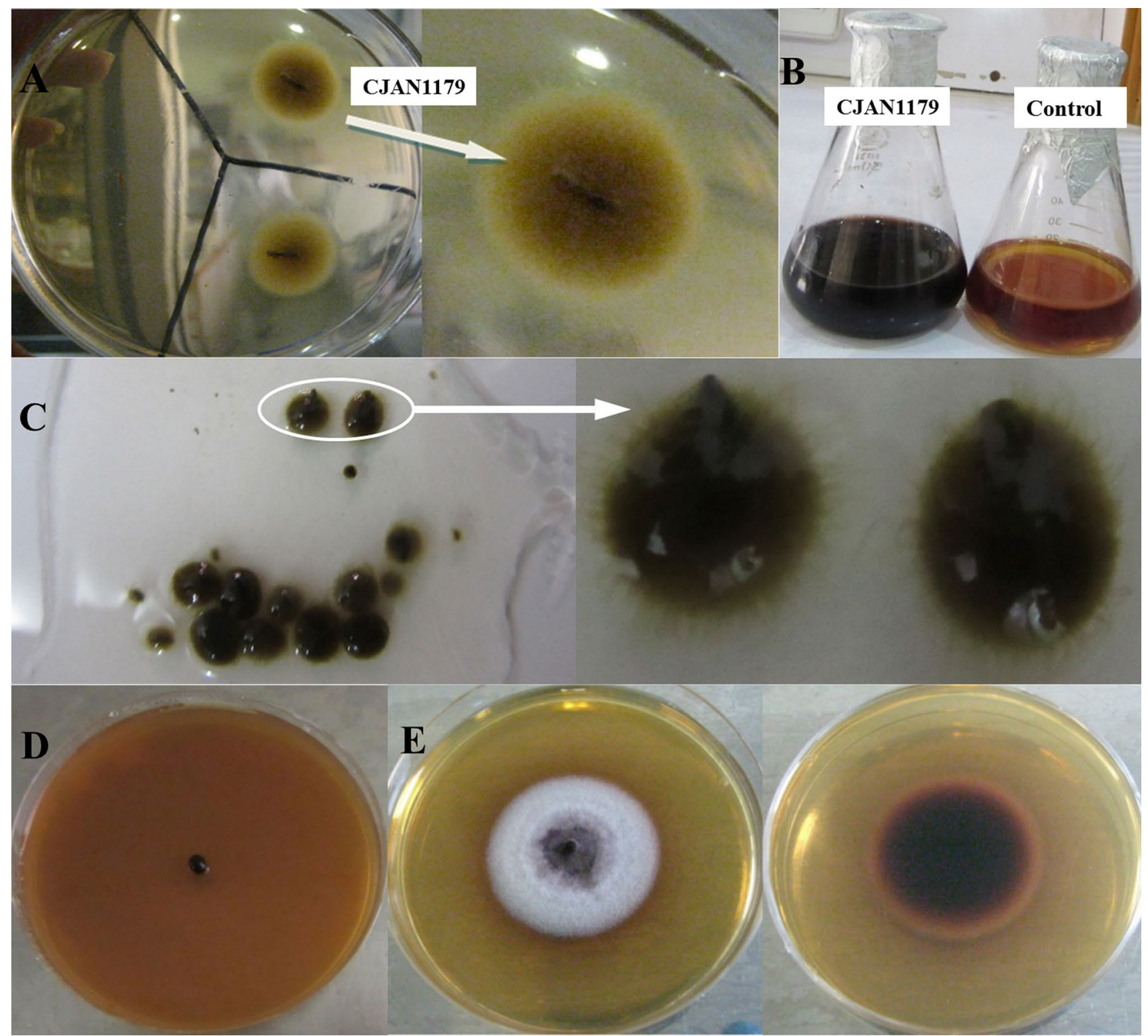

Fig. 5 A Shape of fungus CJAN1179 at the time of isolation from a root piece of $C$. jwarancusa, B cultivation of isolated fungal mycelial colony in SB causes the broth to acquire a deep cherry red colour. C In liquid culture, fungal mycelia turn into smooth and hairy black round sporocarps (fruiting bodies). Fully mature sporocarps are flask- shaped forming beak-like structures called stromata (highlighted on glass slide and shown in zoom). D A single stroma can be cultivated to a fully grown fungal mycelial colony on the agar plate and thus completes and continues the life cycle. E Front and backside view of a fully grown CJAN1179 colony on the agar plate by plants in response to environmental signals and are required in large quantities to maintain normal plant growth under stressful conditions (Waqas et al. 2012). Several bacteria and fungi engaged in plant-microbe interactions have been shown to produce phytohormones such as IAA to improve plant water and nutrient uptake by altering root architecture (Hamayun et al. 2010). Trichoderma species are an example such fungi that have been studied extensively for their potential function in plant growth and survival (Bais et al. 2004; Hermosa et al. 2012). Auxin is known to be responsible for the start and production of lateral roots formed in the post-embryonic stage by several cell divisions because of cell cycle activation in "founder cells" present in the pericycle region, while cytokinins and gibberellins are mostly responsible for shoot growth (Glick 2012). Since environmental stimulations increase auxin production and so enhance lateral root formation, auxin plays a direct role in lateral root formation (Casimiro et al. 2001; Ortíz-Castro et al. 2009). Through auxin and ethylene-related activities, root hair production is also 
linked to the enhancement of plant root architecture for improved water and nutrient uptake from the soil (Masucci and Schiefelbein 1994; Grierson et al. 2014). We hypothesised that CJAN1179 may be exerting its plant growth-promoting effect, directly or indirectly, through one of these phytohormone. Since CJAN1179 leads to an increase of lateral roots, particularly in case of FPI, we assumed that exposure of $A$. thaliana to CJAN1179 either generates the necessary trigger to escalate production of the required phytohormone by the plant or alternatively, CJAN1179 may produce large amounts of the required phytohormone by itself and provides it to the associated plant as a result of fungal-plant interaction. Such comparative effects on the number of lateral roots affected by FPIs and FCFIs have been reported in the literature (Felten et al. 2010).

The naturally occurring prominent auxin, IAA is generated via multiple mechanisms in plants and microorganisms (Gruen 1959; Spaepen and Vanderleyden 2011). The mechanism of auxin production is known only for very few fungi. The major pathway for IAA production in fungi is tryptophan-dependent where tryptophan released from the plant is used by fungi as the main precursor for the production of IAA.

Tryptophan-independent production of IAA has also been reported where indole-3-acetamide (IAM) is converted into IAA (Furukawa et al. 1996; Robinson et al. 1998; Larekeng et al. 2019). Endophytic fungi, pathogen or PGP, are known to produce higher amounts of IAA than free-living fungi (Fouda et al. 2015). IAA has been suggested to serve as a communication signal between the endophytic fungi and the host plant roots (Mehmood et al. 2018; Mehmood et al. 2019) and it is known to affect gene expression in the target organism (Fu et al. 2015). Thus, being an endophyte, we expected CJAN1179 to produce IAA. Our results show that CJAN1179 indeed produces IAA along with some amount of TOL as a by-product of the tryptophan-dependent pathway. This pathway is known to involve indole-3-pyruvic acid (Furukawa et al. 1996). Further, production of excessive lateral roots in the plants exposed to CJAN1179 may also indicate the involvement of volatiles that activate the genes for enzymes related to auxin biosynthesis by the plant although this aspect was not further investigated (Fig. 6). Ethylene and jasmonate, for instance, have been reported as such kind of volatiles (Hermosa et al. 2012). Our results show that this fungus, CJAN1179, is a mutualistic fungus that promotes plant growth by producing IAA, which mediates lateral root growth via a tryptophan-dependent mechanism. Tryptophan, the main precursor of IAA, is secreted by plants during fungal-plant interaction (Felten et al. 2010).

We sequenced the ITS regions of nuclear rRNA for the molecular characterisation of CJAN1179 because the ITS regions in fungal genomes are highly variable among species and even among the entities of the same species of fungi (Liew et al. 1998). This region, therefore, is the source of maximum level of identification among fungal populations (Tarbell 2008; Schoch et al. 2012). CJAN1179 was subjected to molecular characterisation using multiple sequence alignment of the obtained ITS sequence (Suppl. Fig. 3) and phylogenetic
Fig. 6 CJAN1179 auxin production via a tryptophan-depended (TD) mechanism is much higher than by tryptophan-independent (TI) mechanism concluded by current work and displayed in straight line. As described for other fungi, it might be that auxin production by the plant is stimulated by fungal volatiles. This putative fungusplant interaction signalling is displayed in dotted lines and has been proposed for future work. CJAN1179 might produce certain volatiles such as ethylene, jasmonate and sesquiterpenes (SQTs) that activate plant auxin biosynthesis genes. Both auxin production mechanisms are likely involved in enhancing lateral root and root hair formation in A. thaliana. Model adopted and modified from Felten et al. (2010)

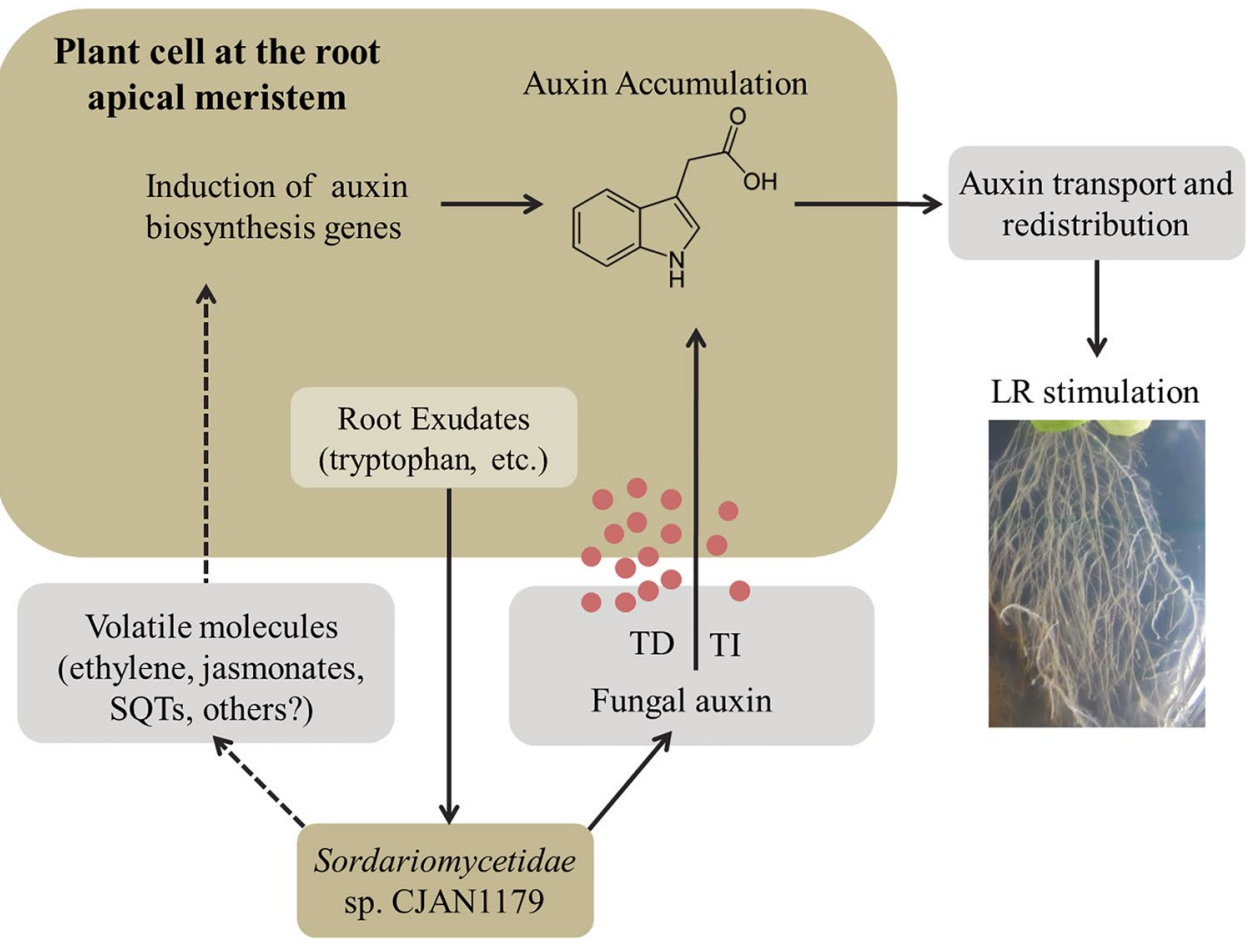


analysis (Fig. 4). CJAN1179 was categorised as a member of the Sordariomycetes. BLAST analysis of our query sequence gave hits with four uncultured fungi in the database. Two of these uncultured fungi with accession numbers GU910815 and GQ924058 have been classified up to order levels, Sordariales and Diaporthales, respectively, while the other two are unclassified. Length of the branches of the two clades, i.e. Magnaporthe oryzae and Magnaporthe grisea, under the order Magnaporthales, clearly separate our query sequence from these and lead to it being categorised in the clade of the uncultured fungi. Based on the maximum sequence similarity with the uncultured Sordariales and Diaporthales isolates we have classified our cultured fungus up to the subclass level, i.e. Sordariomycetidae of class Sordariomycetes, where it possibly represents a new species under the order Sordariales or Diaporthales. The Sordariomycetes are characterised by the production of unitunicate (single-walled), inoperculated (without operculum or lid) asci inside perithecal (flask-shaped) fruiting bodies. Sordariomycetes from different habitats are reported to live as pathogens and endophytes of plants, mammals and arthropods (Zhang et al. 2006; Maharachchikumbura et al. 2015). Our query sequence suggested that CJAN1179 either belongs to the Sordariales or the Diaporthales. Based on background knowledge about Sordariomycetidae (Maharachchikumbura et al. 2016) and macroscopic studies with CJAN1179 showing flask-shaped black sporocarps with beak-like structure, we suggest CJAN1179 to be a member of the order Diaporthales where CJAN1179 appears to be a new species. Thus, according to the phylogenetic analysis following taxonomic lineage can be drawn for CJAN1179: Eukaryota $>$ Fungi $>$ Dikarya $>$ Ascomycota $>$ Pezizomycotina $>$ Sordariomycetes $>$ Sordariomycetidae $>$ Unclassified Sordariomycetidae (probably Diaporthales). The fungus has thus been named as Sordariomycetidae sp. CJAN1179 and the obtained ITS sequence is publicly available (GenBank, accession no. LC198062). On the basis of sequence analysis of CJAN1179 ITS region, we have classified the isolate as a new member of the Sordariomycetidae.

\section{Conclusion}

We demonstrate the role of a root-endophytic fungus CJAN1179 during fungus-plant interaction. The fungus mediates remarkable growth promotion of $A$. thaliana (Col-O) through enhanced lateral root growth. It is capable of producing high amounts of IAA in the presence of tryptophan utilising the tryptophan-dependent indole-3-pyruvate pathway. We believe that during direct fungus-plant interaction, CJAN1179 uses tryptophan secreted by the plant to synthesise IAA which stimulates lateral root formation. The above-mentioned interaction between CJAN1179 and A. thaliana is depicted in a simple hypothetical model (Fig. 6), which was inspired from Felten and co-workers, who studied the beneficial fungus, Laccaria bicolour (Felten et al. 2010).

More research is needed to understand the molecular and physiological elements of the CJAN1179-plant interaction, as well as to look into the fungus' ability to boost plant development in the field. It will also be important to investigate the role this fungus may play in the management of biotic and abiotic stresses in commercially important crops. Information gleaned from such investigations can aid to explore the agricultural potential of arid and wastelands when utilising fungi like CJAN1179.

Supplementary Information The online version contains supplementary material available at https://doi.org/10.1007/s00203-022-02768-2.

Acknowledgements We are grateful to The Cholistan Institute of Desert Studies (CIDS) Bahawalpur, Pakistan for providing us plant samples collected from the Cholistan desert; Yousuf Aziz, Statistics department of The Islamia University of Bahawalpur (IUB) Pakistan for helping us in statistical analysis of the experimental data, and The Molecular Care (MolCare), Molecular Biochemistry Lab, Department of Biochemistry, University of Agriculture, Faisalabad, Pakistan-38040 for phylogenetic analysis using DNA sequence of our query fungus CJAN1179.

Author contributions Submitted study conceived and designed: AN, FHN, FMWG and SS. Project supervision and major lab facilities: FHN (Pakistan), FMWG and SS (Germany). Sample collection facility: MSC. Research performed including material preparation, fungal isolation, fungus-plant interaction studies, biochemical and molecular characterisation of fungi and data collection by: AN. Collaborations and additional lab facilities for biochemical and statistical analysis: MSC and MA. Statistical, phylogenetic and other analysis of the experimental data: AN and FHN. First draft of the manuscript written by: AN. Manuscript reviewed and edited by all the authors. All the authors have approved the version to be published and agree to be accountable for all aspects of the work ensuring that questions related to the accuracy or integrity of any part of the work are appropriately investigated and resolved.

Funding Open Access funding enabled and organized by Projekt DEAL. This work was supported by a research grant (No: 454/R\&D) awarded by the Higher Education Commission (HEC) Pakistan to FHN and a grant under the International Research Support Initiative Program (No: 1-8/HEC/HRD/2014/2973, PIN: IRSIP 25 BMS 06, Dated: February 1st 2014) of HEC Pakistan awarded to AN.

Availability of data and material Supplementary data are being submitted with the manuscript. Further material will be made available if and when required.

Code availability Not applicable.

\section{Declarations}

Conflict of interest None.

Ethical approval Not applicable.

Consent to participate Not applicable. 
Consent for publication The work described in this article is not published previously and is presented in the form agreed and approved by all the authors and responsible institutional authorities where work was carried out, mentioned in this paper; and that, if accepted, any part of the work in this paper will not be submitted anywhere else in the same form without the written consent of the copyright holder of this paper.

Open Access This article is licensed under a Creative Commons Attribution 4.0 International License, which permits use, sharing, adaptation, distribution and reproduction in any medium or format, as long as you give appropriate credit to the original author(s) and the source, provide a link to the Creative Commons licence, and indicate if changes were made. The images or other third party material in this article are included in the article's Creative Commons licence, unless indicated otherwise in a credit line to the material. If material is not included in the article's Creative Commons licence and your intended use is not permitted by statutory regulation or exceeds the permitted use, you will need to obtain permission directly from the copyright holder. To view a copy of this licence, visit http://creativecommons.org/licenses/by/4.0/.

\section{References}

Ahmed M, Hussain M, Dhar MK, Kaul S (2012) Isolation of microbial endophytes from some ethnomedicinal plants of Jammu and Kashmir. J Natl Prod Plant Resour 2:215-220

Aloni R, Aloni E, Langhans M, Ullrich CI (2006) Role of cytokinin and auxin in shaping root architecture: regulating vascular differentiation, lateral root initiation, root apical dominance and root gravitropism. Ann Bot 97:883-893. https://doi.org/10.1093/aob/mc1027

Altschul SF et al (1997) Gapped BLAST and PSI-BLAST: a new generation of protein database search programs. Nucl Acids Res 25:3389-3402. https://doi.org/10.1093/nar/25.17.3389

Arshad M, Hassan AU, Ashraf MY, Noureen S, Moazzam M (2008) Edaphic factors and distribution of vegetation in the Cholistan desert, Pakistan. Pak J Bot 40:1923-1931

Bais HP, Park SW, Weir TL, Callaway RM, Vivanco JM (2004) How plants communicate using the underground information superhighway. Trends Plant Sci 9:26-32. https://doi.org/10.1016/j.tplan ts.2003.11.008

Bhatnagar A, Bhatnagar M (2005) Microbial diversity in desert ecosystems. Curr Sci 89:91-100

Bohlmann H, Wieczorek K (2015) Infection assay of cyst nematodes on Arabidopsis roots. Bio-Protoc 5:1596e. https://doi.org/10.21769/ BioProtoc. 1596

Casimiro I et al (2001) Auxin transport promotes Arabidopsis lateral root initiation. Plant Cell 13:843-852. https://doi.org/10.1105/ tpc. 13.4 .843

Cherif A, Tsiamis G, Compant S, Borin S (2015) BIODESERT: exploring and exploiting the microbial resource of hot and cold deserts. Biomed Res Int 2015:2. https://doi.org/10.1155/2015/289457

Daneshkhah R et al (2013) Piriformospora indica antagonizes cyst nematode infection and development in Arabidopsis roots. J Exp Bot 64:3763-3774. https://doi.org/10.1093/jxb/ert213

Dovana F, Mucciarelli M, Mascarello M, Fusconi A (2015) In Vitro morphogenesis of Arabidopsis to search for novel endophytic fungi modulating plant growth. PLOS ONE 10(12):e0143353. https://doi.org/10.1371/journal.pone.0143353

El-Showk S, Ruonala R, Helariutta Y (2013) Crossing paths: cytokinin signalling and crosstalk. Development 140:1373-1383. https:// doi.org/10.1242/dev.086371
Felsenstein J (2005) PHYLIP (phylogeny inference package) version 3.6. distributed by the author. Department of Genome Sciences. University of Washington, Seattle

Felten J, Legué V, Ditengou FA (2010) Lateral root stimulation in the early interaction between Arabidopsis thaliana and the ectomycorrhizal fungus Laccaria bicolor: Is fungal auxin the trigger? Plant Signal Behav 5:864-867. https://doi.org/10.4161/psb.5.7. 11896

Fouda AH, Hassan SE-D, Eid AM, Ewais EE-D (2015) Biotechnological applications of fungal endophytes associated with medicinal plant Asclepias sinaica (Bioss.). Ann Agric Sci 60:95-104. https://doi.org/10.1016/j.aoas.2015.04.001

Fu S-F, Wei J-Y, Chen H-W, Liu Y-Y, Lu H-Y, Chou J-Y (2015) Indole3-acetic acid: a widespread physiological code in interactions of fungi with other organisms. Plant Signal Behav 10:e1048052. https://doi.org/10.1080/15592324.2015.1048052

Furukawa T, Koga J, Adachi T, Kishi K, Syono K (1996) Efficient conversion of L-tryptophan to Indole-3-acetic acid and/or tryptophol by some species of Rhizoctonia. Plant Cell Physiol 37:899-905. https://doi.org/10.1093/oxfordjournals.pcp.a029037

Glick BR (2012) Plant growth-promoting bacteria: mechanisms and applications. Scientifica 2012:15. https://doi.org/10.6064/2012/ 963401

Glickmann E, Dessaux Y (1995) A critical examination of the specificity of the Salkowski reagent for indolic compounds produced by phytopathogenic bacteria. Appl Environ Microbiol 61:793-796

Gond SK, Verma VC, Mishra A, Kumar A, Kharwar RN (2010) 15 Role of fungal endophytes in plant protection. Management of fungal plant pathogens. CAB International, Wallingford, p 183

Grierson C, Nielsen E, Ketelaarc T, Schiefelbein J (2014) Root hairs. Arabidopsis Book 12:e0172. https://doi.org/10.1199/tab.0172

Gruen HE (1959) Auxins and fungi. Annu Rev Plant Physiol 10:405440. https://doi.org/10.1146/annurev.pp.10.060159.002201

Hamayun M et al (2010) Gibberellin production and plant growth promotion from pure cultures of Cladosporium sp. MH-6 isolated from cucumber (Cucumis sativus L.). Mycologia 102:989-995. https://doi.org/10.3852/09-261

Hameed $\mathrm{M}$ et al (2011) Medicinal flora of the Cholistan desert: a review. Pak J Bot 43:39-50

Hermosa R, Viterbo A, Chet I, Monte E (2012) Plant-beneficial effects of Trichoderma and of its genes. Microbiology 158:17-25. https:// doi.org/10.1099/mic.0.052274-0

Idris EE, Iglesias DJ, Talon M, Borriss R (2007) Tryptophan-dependent production of indole-3-acetic acid (IAA) affects level of plant growth promotion by Bacillus amyloliquefaciens FZB42. Mol Plant Microbe Interact 20:619-626

Kearse M et al (2012) Geneious basic: an integrated and extendable desktop software platform for the organization and analysis of sequence data. Bioinformatics 28:1647-1649. https://doi.org/10. 1093/bioinformatics/bts199

Khan SA, Hamayun M, Khan AL, Lee I-J, Shinwari ZK, Kim J-G (2012) Isolation of plant growth promoting endophytic fungi from dicots inhabiting coastal sand dunes of Korea. Pak J Bot 44:1453-1460

Larekeng SH, Gusmiaty RM, Tunggal A, Susilowati A (2019) Isolation and identification of rhizospheric fungus under Mahoni (Swietenia mahagoni) stands and its ability to produce IAA (Indole Acetid Acid) hormones. IOP Conf Ser 343:012051. https://doi.org/10. 1088/1755-1315/343/1/012051

Liew ECY, Guo LD, Ranghoo VM, Goh TK, Hyde KD (1998) Molecular approaches to assessing fungal diversity in the natural environment. Fungal Divers 1:1-17

Liu D, Coloe S, Baird R, Pedersen J (2000) Rapid mini-preparation of fungal DNA for PCR. J Clin Microbiol 38:471-471

Lugtenberg BJJ, Malfanova N, Kamilova F, Berg G (2013) Plant growth promotion by microbes. In: Bruijn FJD (ed) Molecular 
microbial ecology of the rhizosphere, 1st edn. John Wiley and Sons Inc, Hoboken, pp 561-573

Maharachchikumbura SSN et al (2015) Towards a natural classification and backbone tree for Sordariomycetes. Fungal Divers 72:199301. https://doi.org/10.1007/s13225-015-0331-z

Maharachchikumbura SSN et al (2016) Families of Sordariomycetes. Fungal Divers 79:1-317. https://doi.org/10.1007/ s13225-016-0369-6

Makhalanyane TP, Valverde A, Gunnigle E, Frossard A, Ramond J-B, Cowan DA (2015) Microbial ecology of hot desert edaphic systems. FEMS Microbiol Rev 39:203-221. https://doi.org/10.1093/ femsre/fuu011

Maor R, Haskin S, Levi-Kedmi H, Sharon A (2004) In planta production of indole-3-acetic acid by Colletotrichum gloeosporioides $\mathrm{f}$. sp. aeschynomene. Appl Environ Microbiol 70:1852-1854

Masucci JD, Schiefelbein JW (1994) The rhd6 mutation of Arabidopsis thaliana alters root-hair initiation through an auxin- and ethyleneassociated process. Plant Physiol 106:1335-1346. https://doi.org/ 10.1104/pp.106.4.1335

Mehmood A et al (2018) IAA producing endopytic fungus Fusariun oxysporum wlw colonize maize roots and promoted maize growth under hydroponic condition. Eur J Exp Biol. https://doi.org/10. 21767/2248-9215.100065

Mehmood A, Hussain A, Irshad M, Hamayun M, Iqbal A, Khan N (2019) In vitro production of IAA by endophytic fungus Aspergillus awamori and its growth promoting activities in Zea mays. Symbiosis 77:225-235. https://doi.org/10.1007/ s13199-018-0583-y

Mei C, Flinn BS (2010) The use of beneficial microbial endophytes for plant biomass and stress tolerance improvement. Recent Pat Biotechnol 4:81-95. https://doi.org/10.2174/187220810790069523

Mohite B (2013) Isolation and characterization of indole acetic acid (IAA) producing bacteria from rhizospheric soil and its effect on plant growth. J Soil Sci Plant Nutr 13:638-649

Morgan JAW, Bending GD, White PJ (2005) Biological costs and benefits to plant-microbe interactions in the rhizosphere. J Exp Bot 56:1729-1739. https://doi.org/10.1093/jxb/eri205

Nasim FH, Naureen A, Saleem M, Riaz N, Choudhary MS, Ashraf M (2018) PAAN135, a novel rhizospheric fungus associated with Cholistan desert grass Panicum antidotale, is a species of Saccharomycetales and a new source of cyclo-L-prolylglycine diketopiperazine. Symbiosis 74:121-130. https://doi.org/10.1007/ s13199-017-0495-2

Nei M, Kumar S (2000) Molecular evolution and phylogenetics. Oxford University Press Inc, New York

Ortíz-Castro R, Contreras-Cornejo HA, Macías-Rodrígueza L, LópezBucio J (2009) The role of microbial signals in plant growth and development. Plant Signal Behav 4:701-712. https://doi.org/10. 4161/psb.4.8.9047

Rafay M, Khan RA, Yaqoob S, Ahmad M (2013) Floristic composition of grass species in the degrading rangelands of Cholistan desert. Pak J Agric Sci 50:599-603

Rahman A, Sitepu IR, Tang S-Y, Hashidoko Y (2010) Salkowski's reagent test as a primary screening index for functionalities of rhizobacteria isolated from wild dipterocarp saplings growing naturally on medium-strongly acidic tropical peat soil. Biosci Biotechnol Biochem 74:2202-2208. https://doi.org/10.1271/bbb.100360

Rehman F, Hussain T, Abdullah M, Ashraf I, Rafay M, Bibi I (2015) Ethnobotanical survey; common medical plants used by people of Cholistan desert. Prof Med J 22:1367-1372. https://doi.org/10. 17957/TPMJ/15.2880
Robinson M, Riov J, Sharon A (1998) Indole-3-acetic acid biosynthesis in Colletotrichum gloeosporioides f. sp.aeschynomene. Appl Environ Microbiol 64:5030-5032

Rodriguez RJ, Redman RS, Henson JM (2004) The role of fungal symbioses in the adaptation of plants to high stress environments. Mitig Adapt Strat Glob Change 9:261-272. https://doi.org/10. 1023/b:miti.0000029922.31110.97

Rodriguez RJ, White JF Jr, Arnold AE, Redman RS (2009) Fungal endophytes: diversity and functional roles. New Phytol 182:314330. https://doi.org/10.1111/j.1469-8137.2009.02773.x

Saitou N, Nei M (1987) The neighbor-joining method: a new method for reconstructing phylogenetic trees. Mol Biol Evol 4:406-425. https://doi.org/10.1093/oxfordjournals.molbev.a040454

Sarwar M, Kremer RJ (1995) Determination of bacterially derived auxins using a microplate method. Lett Appl Microbiol 20:282-285. https://doi.org/10.1111/j.1472-765X.1995.tb00446.x

Schoch CL et al (2012) Nuclear ribosomal internal transcribed spacer (ITS) region as a universal DNA barcode marker for Fungi. Proc Natl Acad Sci 109:6241-6246. https://doi.org/10.1073/pnas. 1117018109

Sijmons PC, Grundler FMW, Mende N, Burrows PR, Wyss U (1991) Arabidopsis thaliana as a new model host for plant-parasitic nematodes. Plant J 1:245-254. https://doi.org/10.1111/j.1365-313X. 1991.00245.x

Spaepen S, Vanderleyden J (2011) Auxin and plant-microbe interactions. Cold Spring Harbor Perspect Biol 3:a001438

Sun J et al (2009) Arabidopsis ASAl is important for jasmonate-mediated regulation of auxin biosynthesis and transport during lateral root formation. Plant Cell 21:1495-1511. https://doi.org/10.1105/ tpc.108.064303

Tamura K, Peterson D, Peterson N, Stecher G, Nei M, Kumar S (2011) MEGA5: molecular evolutionary genetics analysis using maximum likelihood, evolutionary distance, and maximum parsimony methods. Mol Biol Evol 28:2731-2739. https://doi.org/10.1093/ molbev/msr121

Tarbell T (2008) Molecular characterization of fungal species from pure cultures and environmental samples. Department of biological sciences. Fordham University, New York, p 15

Waqas M et al (2012) Endophytic fungi produce gibberellins and indoleacetic acid and promotes host-plant growth during stress. Molecules 17:10754-10773. https://doi.org/10.3390/molecules1 70910754

Zhang N et al (2006) An overview of the systematics of the Sordariomycetes based on a four-gene phylogeny. Mycologia 98:10761087. https://doi.org/10.1080/15572536.2006.11832635

Zhou Z et al (2014) Diversity and plant growth-promoting ability of endophytic fungi from the five flower plant species collected from Yunnan, Southwest China. J Plant Interact 9:585-591. https://doi. org/10.1080/17429145.2013.873959

Zuo X et al (2015) Plant functional diversity enhances associations of soil fungal diversity with vegetation and soil in the restoration of semiarid sandy grassland. Ecol Evol 6:318-328. https://doi.org/ 10.1002/ece3.1875

Publisher's Note Springer Nature remains neutral with regard to jurisdictional claims in published maps and institutional affiliations. 